\title{
Analysis of Solution Quality of a Multiobjective Optimization-based Evolutionary Algorithm for Knapsack Problem
}

\author{
Jun He, Yong Wang and Yuren Zhou
}

\begin{abstract}
Multi-objective optimisation is regarded as one of the most promising ways for dealing with constrained optimisation problems in evolutionary optimisation. This paper presents a theoretical investigation of a multi-objective optimisation evolutionary algorithm for solving the 0-1 knapsack problem. Two initialisation methods are considered in the algorithm: local search initialisation and greedy search initialisation. Then the solution quality of the algorithm is analysed in terms of the approximation ratio.
\end{abstract}

\section{INTRODUCTION}

Consider the problem of maximizing an objective function,

$$
\max _{\vec{x}} f(\vec{x}), \quad \text { subject to } g(x) \leq 0 .
$$

The above constrained optimisation problem can be transferred into an unconstrained bi-objective optimisation problem. That is to optimize the original objective function plus to minimize the constraint violation simultaneously:

$$
\left\{\begin{array}{l}
\max _{\vec{x}} f(\vec{x}), \\
\min _{\vec{x}} v(\vec{x})
\end{array}\right.
$$

where $v(\vec{x})$ is the degree of constraint violation, given by

$$
v(\vec{x})= \begin{cases}0, & \text { if } g(\vec{x}) \leq 0 \\ g(\vec{x}), & \text { otherwsie }\end{cases}
$$

The use of multi-objectives for single-objective optimisation problems could be traced back to 1990s [1]. This methodology has been termed multiobjectivisation [2]. Using multiobjectivization sometimes may help the search more efficient as shown in $[3]-[6]$.

According to the survey [7], multi-objective optimisation is regarded as one of the most promising ways for dealing with constrained optimisation problems in evolutionary optimisation. A constrained optimisation problem is often transformed into a bi-objective optimisation problem, in which the first objective is the original objective function and the second objective is the degree of constraint violation [8]-[11]. After this transformation, Pareto dominance is frequently employed to compare individuals. Currently the research in this area is very active [7]. For example, a self-adaptive selection method is proposed recently in [12], which aims to exploit both non-dominated solutions with low constraint violations and feasible solutions with low objective function values. Multi-objective optimisation is combined with differential evolution in [13] and an infeasible solution replacement mechanism is proposed. A dynamic hybrid framework is presented in [14], where the global and local search models are implemented dynamically according to the feasibility proportion of the population.

This paper aims at analysing the solution quality of evolutionary algorithms (EAs) in terms of the approximation ratio. It is not intended to demonstrate that EAs are able to compete with problem-specific approximation algorithms, since this is unlikely in most cases. Nevertheless, it is still necessary and important to understand the solution quality of EAs, so the EAs with arbitrarily bad solution quality could be avoided in applications. The analysis of the approximation performance of EAs has attracted a lot of interests in recent years [15]-[17].

This paper investigate an existing multiobjective optimization-based EA [9] (MOEA) for solving constrained optimisation problems. The MOEA originally is designed for continuous optimization. Here it is adapted for solving the 0-1 knapsack problem. Although experiment results show its performance is good, no theoretical analysis exists for this MOEA [9]. This motivates our rigorous analysis.

The remainder of the paper is organized as follows. The 0-1 knapsack problem and approximation ratio are introduced in Section [II The MOEA with the local search initialisation is analysed in Section III. Section IV is devoted to the analysis of the MOEA with the greedy search initialisation. Section $\nabla$ concludes the article.

This work was partially supported by EPSRC under Grant No. EP/I009809/1 (He), by NSFC under Grant Nos. 61170081, 61472143 (Zhou), 61273314 and by the Program for New Century Excellent Talents in University under Grant NCET-13-0596 (Wang).

Jun He is with Department of Computer Science, Aberystwyth University, Aberystwyth, UK

Yong Wang is with School of Information Science and Engineering, Central South University, Changsha 410083, China

Yuren Zhou is with School of Advanced Computing, Sun Yat-sen University, Guangzhou, 510006, China 


\section{0-1 KnApsack Problem and Approximation Ratio of Solutions}

Given an instance of the 0-1 knapsack problem with a set of weights $w_{i}$, values $v_{i}$, and capacity $W$ of a knapsack, the task is to find a binary string $\vec{x}_{\max }$ so as to maximize the objective function,

$$
\max _{\vec{x}} f(\vec{x})=\sum_{i=1}^{n} v_{i} x_{i}, \quad \text { subject to } \sum_{i=1}^{n} w_{i} x_{i} \leq W,
$$

where $\vec{x}=\left(x_{1} \cdots x_{n}\right)$ is a binary string. $x_{i}=1$ if item $i$ is selected in the knapsack; otherwise $x_{i}=0$.

A feasible solution is a knapsack represented by an $\vec{x}$ which satisfies the constraint, that is $\sum_{i=1}^{n} w_{i} x_{i} \leq W$. An infeasible one is an $\vec{x}$ that violates the constraint. The string $(0 \ldots 0)$ represents a null knapsack. Without loss of generality, assume that a feasible solution always exists and $n$ is large.

There exist well-known approximation algorithms for the 0-1 knapsack problem [18], [19]. Probably the simplest one is the greedy search [18] whose worst-case approximation performance ratio equals to $1 / 2$ and time complexity is $O(n)$ plus $O(n \log n)$ for the initial sorting. A polynomial-time approximation scheme has been introduced in [18] whose worse-case performance is $k /(1+k)$ given an integer parameter $k$ and its time complexity is $O\left(n^{k+1}\right)$. Furthermore, a fully-polynomialtime approximation scheme is well-known [18] whose time complexity is $O\left(n / \epsilon^{2}\right)$ plus $O(n \log n)$ for the initial sorting given a parameter $\epsilon>0$.

In evolutionary optimisation, the 0-1 knapsack problem has been taken as a benchmark in computer experiments [20], [21] for evaluating the performance of various constraint-handling techniques. It is also one of favourite problems used in the theoretical study of EAs [22], [23].

In order to assess the solution quality of an EA, an evolutionary approximation algorithm is defined as below. It follows the definition of conventional $\alpha$-approximation algorithms [24, Definition 1].

Definition 1: An EA is an $\alpha$-approximation algorithm for a constrained optimisation problem if for all instances of the problem, the EA can produce a feasible solution in polynomial running time, whose objective function value is within a factor of $\alpha$ of that of an optimal solution. The running time of an EA is the expected number of function evaluations.

In a maximisation problem (assume $f\left(\vec{x}_{\max }\right)>0$ ), a feasible solution $\vec{x}$ is called to have an $\alpha$-approximation ratio if it satisfies

$$
\frac{f(\vec{x})}{f\left(\vec{x}_{\max }\right)} \geq \alpha \text {. }
$$

In order to prove that an EA is not an $\alpha$-approximation algorithm, it is sufficient to show that an EA needs exponential running time to obtain a feasible solution with an $\alpha$-approximation ratio in one instance of the problem.

\section{ANAlysis of MOEA With LOCAL SEARCH INITIALISATION}

The 0-1 knapsack problem can be transformed into a bi-objective optimisation problem, that is to maximize the objective function $f(\vec{x})$ and to minimize the constrain violation $v(\vec{x})$, where $v(\mathbf{x})$ is defined by

$$
v(\mathbf{x})= \begin{cases}0 ; & \text { if } \mathbf{x} \text { is feasible } \\ \sum_{i=1}^{n} w_{i}-W, & \text { otherwise }\end{cases}
$$

Although a constrained optimisation problems can be converted into a bi-objective optimisation problem, there exists an essential difference between it and general multi-objective optimisation problems [9]. The target of general multi-objective optimisation is to obtain a final population with a diverse non-dominated individuals uniformly distributed on the Pareto front. However in the bi-objective optimisation problem derived from contained optimisation, the target is to obtain the optimal feasible solution of the original constrained optimisation problem. Consequently, there is no need to care about the uniform distribution of the resulting solutions on the Pareto front.

The MOEA adopted in this section is a variant of an existing MOEA proposed in [9]. The fundamental idea in this MOEA is that non-dominated individuals in a children population are chosen and replace dominated individuals of the parent population. The algorithm, based on Model 1 of [9], is described in Algorithm 1 for solving the 0-1 knapsack problem.

It should be pointed out that the running time of EAs is dependent on initialisation. Two initialisation methods are considered in the paper: initialisation by the local search and by the greedy search. In this section, we investigate the first one: the local search initialisation, described in Algorithm 2. This initialisation does not only produce both feasible solutions (local optima), but also infeasible solutions. Bitwise mutation flips each bit of a binary string with probability $\frac{1}{n}$. Population size $N$ is set to a large constant and for the sake of analysis, assume that $N / 4$ is an integer.

We show that the solution quality of the MOEA with the local search initialisation might be arbitrarily bad using the following instance of the 0-1 knapsack problem.

Instance 1: In the following table, $H, I$ and $J$ represent index sets. For the sake of simplicity, assume that $\frac{n}{2}$ and $\frac{\alpha n}{2}$ are integers. Fixing a constant $\alpha \in(0,1)$, choose $n$ an enough large integer so that $n>\frac{2}{\alpha}$ and $\frac{2}{\alpha}>\frac{n \alpha^{2} \ln n}{2}$. 

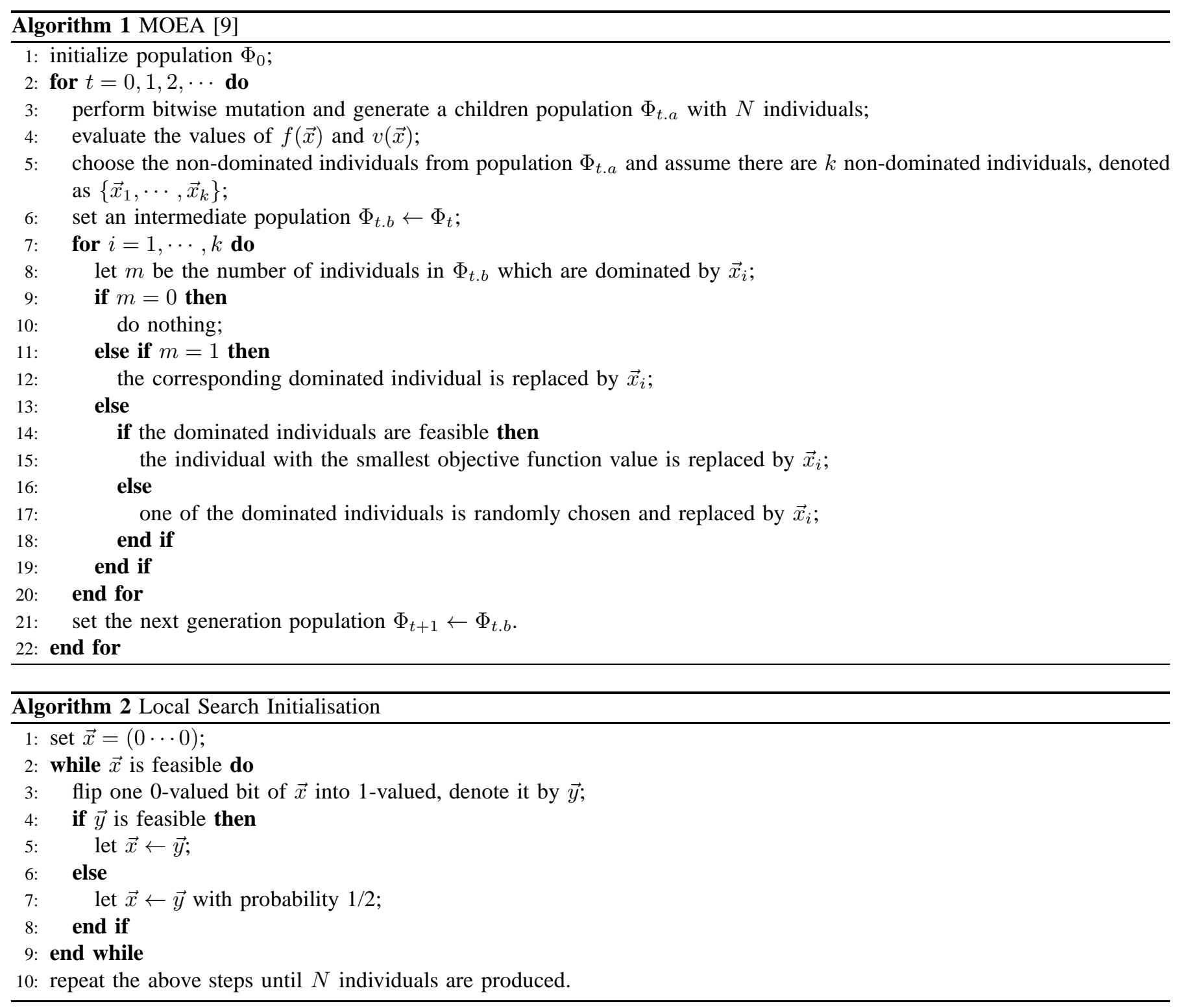

Let $\vec{x}_{\text {max }}$ represent the global optimum such that $x_{1}=1$ and other bits $x_{i}=0$. The global optimum is unique. Its objective function value is

$$
f\left(\vec{x}_{\max }\right)=n \text {. }
$$

Let $\vec{x}_{\text {loc }}$ represent a local optimum 1 such that $\frac{\alpha n}{2}$ bits $x_{i}=1$ (where $i \in I$ ) and other bits $\vec{x}_{i}=0$. Its objective function value is

$$
f\left(\vec{x}_{\text {loc }}\right)=\frac{\alpha n}{2} .
$$

$f\left(\vec{x}_{\text {loc }}\right)$ is the second largest objective function value among feasible solutions. The number of such local optima $\vec{x}_{\text {loc }}$ is experiential in $n$,

$$
\left(\begin{array}{c}
\frac{n}{2} \\
\frac{\alpha n}{2}
\end{array}\right) \geq\left(\frac{1}{\alpha}\right)^{\frac{\alpha n}{2}}
$$

Let $\vec{x}_{\mathrm{vio} 1}$ denote an infeasible solution such that $x_{i}=1$ for $\frac{\alpha n}{2}$ indexes $i \in I$, one $i \in J$, and $x_{i}=0$ for other $i$. Its objective function value and violation value are

$$
f\left(\vec{x}_{\mathrm{vio} 1}\right)=f\left(\vec{x}_{\mathrm{loc}}\right)+\alpha^{\ln n}, \quad v\left(\vec{x}_{\mathrm{vio} 1}\right)=\alpha^{2 \ln n} .
$$

${ }^{1}$ A feasible solution $\vec{x}$ is called a local optimum if $f(\vec{y})<f(\vec{x})$ for any feasible solution $\vec{y}$ within Hamming distance $d(\vec{x}, \vec{y})=1$. 


\begin{tabular}{c|c|c|c}
\hline & $H$ & $I$ & $J$ \\
\hline$i$ & 1 & $2, \cdots, \frac{n}{2}+1$ & $\frac{n}{2}+2, \cdots, n$ \\
\hline$v_{i}$ & $n$ & 1 & $\alpha^{\ln n}$ \\
$w_{i}$ & $n$ & $\frac{2}{\alpha}$ & $\alpha^{2 \ln n}$ \\
\hline$W$ & \multicolumn{3}{|c}{$n$} \\
\hline \multicolumn{3}{c}{ TABLE I } \\
\hline \multicolumn{3}{c}{ INSTANCE I }
\end{tabular}

The number of such infeasible solutions $\vec{x}_{\mathrm{vio} 1}$ is exponential in $n$,

$$
\left(\begin{array}{c}
\frac{n}{2} \\
\frac{\alpha n}{2}
\end{array}\right) \frac{n-1}{2} \geq \frac{n-1}{2}\left(\frac{1}{\alpha}\right)^{\frac{\alpha n}{2}} .
$$

Let $\vec{x}_{\mathrm{vio2}}$ denote an infeasible solution such that $x_{1}=1, x_{i}=1$ for one $i \in J$, and $x_{i}=0$ for any other $i$. Its objective function value and violation value are

$$
f\left(\vec{x}_{\mathrm{vio} 2}\right)=f\left(\vec{x}_{\max }\right)+\alpha^{\ln n}, \quad v\left(\vec{x}_{\mathrm{vio} 2}\right)=\alpha^{2 \ln n} .
$$

It is easy to verify that the degree of constraint violation $v\left(\vec{x}_{\mathrm{vio} 1}\right)$ and $v\left(\vec{x}_{\mathrm{vio} 2}\right)\left(=\alpha^{\ln n}\right)$ is the minimum among all infeasible solutions.

Instance 1 is hard since Hamming distance between a local optimum $\vec{x}_{\text {loc }}$ and the unique global optimum $\vec{x}_{\max }$ is large $\geq \alpha n / 2$ and the the number of local optima is exponential in $n$. Since the selection used in the MOEA is that non-dominated individuals in a children population are chosen and replace dominated individuals of the parent population, it prevents individuals moving from the 2nd best fitness level to the best fitness level. Thus the EA needs exponential time to leave the absorbing basin of the local optima.

Theorem 1: For Instance 1 and any constant $\alpha \in(0,1)$, the MOEA with the local search initialisation needs $\Omega\left(n^{\frac{\alpha n}{2}}\right)$ running time to find an $\alpha$-approximation solution in the worst case.

Proof: After the initialisation, individuals generated by the local search may include $\vec{x}_{\max }$, local optima $\vec{x}_{\mathrm{loc}}$ and infeasible solutions $\vec{x}$ with Hamming distance $H\left(\vec{x}, \vec{x}_{\max }\right)=1$ or $H\left(\vec{x}, \vec{x}_{\mathrm{loc}}\right)=1$. The worst case is that after initialisation, population $\Phi_{0}$ is composed of $N$ local optima $\vec{x}_{\text {loc }}$ and infeasible solutions $\vec{x}_{\text {viol }}$. Notice that the number of local optima $\vec{x}_{\text {loc }}$ and infeasible solutions $\vec{x}_{\text {vio1 }}$ is exponential in $n$. The individuals in $\Phi_{0}$ may be chosen to be different.

Assume that in the $t$ th generation, population $\Phi_{t}$ is composed of $N$ local optima $\vec{x}_{\text {loc }}$ and infeasible solutions $\vec{x}_{\text {vio1 }}$. The approximation ratio between $f\left(\vec{x}_{\text {loc }}\right)$ and $f\left(\vec{x}_{\max }\right)$ is

$$
\frac{f\left(\vec{x}_{\mathrm{loc}}\right)}{f\left(\vec{x}_{\max }\right)}=\frac{\alpha}{2}<\alpha .
$$

Since $\alpha$ could be any constant, the above approximation ratio could arbitrarily bad.

As we know that $\vec{x}_{\max }$ is the unique solution satisfying $f\left(\vec{x}_{\max }\right)>f\left(\vec{x}_{\text {loc }}\right)$, it is sufficient to prove that the EA needs exponential running time to generate $\vec{x}_{\max }$.

First we consider the event of mutating $\vec{x}_{\text {loc }}$ or $\vec{x}_{\text {viol }}$ into a child $\vec{y}$. The event can be decomposed into the following mutually exclusive and exhaustive sub-events.

1) $\vec{y}$ is a feasible solution such that $f(\vec{y})<f\left(\vec{x}_{\text {loc }}\right)$.

Obviously $\vec{y}$ will not dominate $\vec{x}_{\text {loc }}$. At the same time, $\vec{y}$ will not dominate $\vec{x}_{\text {vio1 }}$ since $f(\vec{y})<f\left(\vec{x}_{\text {loc }}\right)<f\left(\vec{x}_{\text {vio } 1}\right)$. Thus $\vec{y}$ will not dominate any individuals in $\Phi_{t}$ and cannot be selected into the next generation population.

2) $\vec{y}$ is a feasible solution such that $f(\vec{y})=f\left(\vec{x}_{\text {loc }}\right)$, that is, $\vec{y}$ is a $\vec{x}_{\text {loc }}$.

3) $\vec{y}$ is a feasible solution such that $f(\vec{y})>f\left(\vec{x}_{\text {loc }}\right)$, that is, $\vec{y}$ is the global optimum $\vec{x}_{\text {max }}$.

In this case, all 1 -valued bits $x_{i}$ (where $i \in I$ ) must be flipped from 1 to 0 . The probability of the event happening is at most

$$
O\left(\frac{1}{n}\right)^{\frac{\alpha n}{2}} .
$$

4) $\vec{y}$ is an infeasible solution such that $v(\vec{y})>v\left(\vec{x}_{\text {vio1 }}\right)$.

In this case, $\vec{y}$ will not dominate $\vec{x}_{\text {loc }}$ and $\vec{x}_{\text {vio } 1}$, so it cannot be selected into the next generation population.

5) $\vec{y}$ is an infeasible solution such that $v(\vec{y})=v\left(\vec{x}_{\mathrm{vio} 1}\right)$, that implies,

a) either $\vec{y}$ is $\vec{x}_{\text {viol }}$;

b) or $\vec{y}$ is an infeasible solution $\vec{x}_{\text {vio2 }}$.

In the second case, all 1 -valued bits $x_{i}$ (where $i \in I$ ) must be flipped from 1 to 0 . The probability of the event happening is

$$
O\left(\frac{1}{n}\right)^{\frac{\alpha n}{2}}
$$


From the above analysis, we observe that only when either $\vec{x}_{\max }$ or an infeasible solution $\vec{x}_{\text {vio2 }}$ is generated via mutation, the population status could be changed. Otherwise the population is still composed of $N$ solutions $\vec{x}_{\text {loc }}$ and $\vec{x}_{\text {viol }}$. The probability of the former event happening is $O\left(\frac{1}{n}\right)^{\frac{\alpha n}{2}}$.

Next we analyse the role of using a population. Consider the event that a population includes either $\vec{x}_{\text {max }}$ or an infeasible solution $\vec{x}_{\text {vio2 }}$ is generated via mutation. Since $N$ parents are selected and mutated independently, the probability of the event happening is $N O\left(n^{\frac{2}{\alpha n}}\right)$. Thus the expected number of generations for the EA to reach $\vec{x}_{\max }$ is $\frac{1}{N} \Omega\left(n^{\frac{\alpha n}{2}}\right)$. Since there are $N$ fitness evaluations at each generation, the expected number of fitness evaluations is $\Omega\left(n^{\frac{\alpha n}{2}}\right)$. The required conclusion is then proven.

\section{Analysis of MOEA with GREedy SEARCH INITIALISATION}

In order to produce a good quality solution with a guaranteed approximation ratio, a natural idea is to combine an EA with an approximation algorithm: first we apply an approximation algorithm to producing approximation solutions as the initial population, and then apply the MOEA to searching a global optimum. In this section, we consider a $\frac{1}{2}$-approximation algorithm to implement the initialisation. It a variant of the greedy search [18, Section 2.4], described in Algorithm 3 . Notice that the initialisation does not only produce feasible solutions (local optima), but also infeasible solutions.

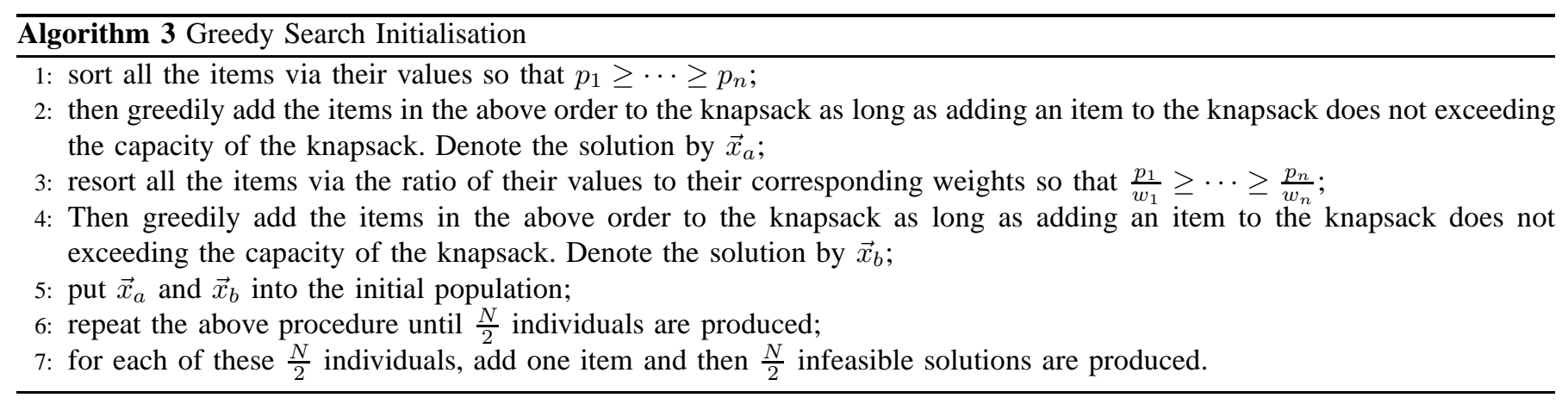

Using the greedy search initialisation, we may find the global optimal solution of Instance 1 during the initialisation phase. Furthermore, since the greedy search is a 1/2-approximation algorithm for the $0-1$ knapsack problem, the MOEA with the greedy search initialisation is an evolutionary $1 / 2$-approximation algorithm too. The advantage of using an EA is the ability to obtain the global optimum due to the use of bitwise mutation.

In the following we answer the question: can the MOEA with the greedy search initialisation find a solution with the approximation ratio better than 1/2? Through analysing the instance described below, we obtain a negative answer.

Instance 2: In the following table, $H, I, J$ and $K$ represent index sets. For the sake of simplicity, assume that $n / 4$ is an integer.

\begin{tabular}{c|c|c|c|c}
\hline & $H$ & $I$ & $J$ & $K$ \\
\hline$i$ & 1,2 & $3, \cdots, \frac{n}{4}$ & $\frac{n}{4}+1, \cdots, \frac{n}{2}$ & $\frac{n}{2}+1, \cdots, n$ \\
\hline$v_{i}$ & $n$ & $n+2$ & $n^{-3}$ & $n^{-3}$ \\
$w_{i}$ & $n$ & $n+1$ & $n^{-4}$ & $\frac{1}{4\left(1+n^{-4}\right)}$ \\
\hline$W$ & \multicolumn{4}{c}{ TABLE II } \\
\hline \multicolumn{5}{c}{ INSTANCE II }
\end{tabular}

Let $\vec{x}_{\max }$ represent the unique global optimum such that $x_{i}=1$ for any $i \in H$ and $x_{i}=0$ for any other $i$. Its objective function value is

$$
f\left(\vec{x}_{\max }\right)=2 n \text {. }
$$

Let $\vec{x}_{\text {loc }}$ represent a local optimum such that $x_{i}=1$ for one $i \in I$, any $i \in J$ and $\frac{n}{4}-2$ indexes $i \in K ; x_{i}=0$ for all other $i$. Its objective function value is

$$
f\left(\vec{x}_{\text {loc }}\right)=n+2+\left(\frac{n}{2}-2\right) n^{-3} .
$$

$f\left(\vec{x}_{\text {loc }}\right)$ is the second largest objective function value among feasible solutions.

Let $\vec{x}_{\text {vio1 }}$ represent an infeasible solution such that $x_{i}=1$ for one $i \in I$, any $i \in J$ and $\frac{n}{4}-1$ indexes $i \in K$; $x_{i}=0$ for all other $i$. Its objective function value and violation value are

$$
f\left(\vec{x}_{\mathrm{vio} 1}\right)=f\left(\vec{x}_{\mathrm{loc}}\right)+n^{-3}, \quad v\left(\vec{x}_{\mathrm{vio} 1}\right)=\frac{1}{4\left(1+n^{-4}\right)} .
$$


Let $\vec{x}_{\text {vio2 }}$ represent an infeasible solution such that $x_{i}=1$ for any $i \in H$ and one $i \in K$, and $x_{i}=0$ for any other $i$. Its objective function value and violation value satisfy

$$
f\left(\vec{x}_{\mathrm{vio} 2}\right)=f\left(\vec{x}_{\max }\right)+n^{-3}, \quad v\left(\vec{x}_{\mathrm{vio} 2}\right)=\frac{1}{4\left(1+n^{-4}\right)} .
$$

Let $\vec{x}_{\text {vio3 }}$ represent an infeasible solution such that $x_{i}=1$ for any $i \in H$ and at least one $i \in J$, and $x_{i}=0$ for any other $i$. Its objective function value and violation value satisfy

$$
f\left(\vec{x}_{\max }\right)<f\left(\vec{x}_{\text {vio3 }}\right) \leq f\left(\vec{x}_{\max }\right)+\frac{n^{-2}}{4}, \quad 0<v\left(\vec{x}_{\text {vio3 }}\right)<\frac{n^{-3}}{4} .
$$

Instance 2 is a hard problem to EAs using bitwise mutation since Hamming distance between a local optimum $\vec{x}_{\text {loc }}$ and the unique global optimum $\vec{x}_{\max }$ is large. Another trouble is the number of local optima which is exponential in $n$. Thus it is difficult for a population to leave the absorbing basin of the local optima.

Theorem 2: For Instance 2, the MOEA with the greedy search initialisation can find a $\frac{1}{2}$-approximation solution after initialisation. But in the worst case, it needs $\Omega\left(n^{\frac{n}{4}}\right)$ running time to find a $\left(\frac{1}{2}+\frac{1}{n}+\frac{1}{2 n^{3}}\right)$-approximation solution.

Proof: The first conclusion is trivial due to the use of the greedy search. After the initialisation, individuals generated by the greedy search are local optima $\vec{x}_{\text {loc }}$ and infeasible solutions $\vec{x}$ with Hamming distance $H\left(\vec{x}, \vec{x}_{\text {loc }}\right)=1$. The local optimum $\vec{x}_{\text {loc }}$ has an approximation ratio given by

$$
\frac{f\left(\vec{x}_{\mathrm{loc}}\right)}{f\left(\vec{x}_{\max }\right)}=\frac{n+2+\left(\frac{n}{2}-2\right) n^{-3}}{2 n} \in\left(\frac{1}{2}, \frac{1}{2}+\frac{1}{n}+\frac{1}{2 n^{3}}\right) .
$$

The proof of the second conclusion is similar to that of Theorem 1 . The worst case is that after initialisation, population $\Phi_{0}$

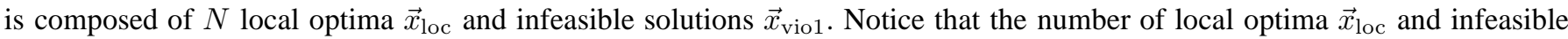
solutions $\vec{x}_{\text {vio1 }}$ is exponential in $n$. The individuals in $\Phi_{0}$ could be chosen to be different.

Assume that in the $t$ th generation, population $\Phi_{t}$ is composed of $N$ different local optima $\vec{x}_{\text {loc }}$ and infeasible solutions $\vec{x}_{\text {viol }}$. Let $\mathbf{y}$ be a child mutated from a parent $\mathbf{x}$. The event can be decomposed into the following mutually exclusive and exhaustive sub-events.

1) $\mathbf{y}$ is feasible such that $f(\mathbf{y})<f\left(\mathbf{x}_{\text {loc }}\right)$.

According to the selection based on the Pareto-dominance, $\mathbf{y}$ will not be selected to the next generation population.

2) $\mathbf{y}$ is feasible such that $f(\mathbf{y})=f\left(\mathbf{x}_{\text {loc }}\right)$, that is, $\mathbf{y}$ is a local optimum $\vec{x}_{\text {loc }}$.

3) $\mathbf{y}$ is feasible such that $f(\mathbf{y})>f\left(\mathbf{x}_{\text {loc }}\right)$, that is, $\vec{y}$ is $\vec{x}_{\max }$.

In this case, any 1 -valued bit $x_{i}$ where $i \in I \cup J \cup K$ must be flipped from 1 to 0 , and $x_{1}$ and $x_{2}$ must be flipped from 0 to 1 . Thus at least $\frac{n}{4}$ bits must be flipped. The probability of this event happening is at most $O\left(n^{-\frac{n}{4}}\right)$.

4) $\mathbf{y}$ is infeasible such that $v(\mathbf{x})=\frac{1}{4\left(1+n^{-4}\right)}$, that is

- either $\mathbf{y}$ is $\vec{x}_{\mathrm{vio} 1}$;

- or $\mathbf{y}$ is $\vec{x}_{\mathrm{vio} 2}$.

In the second case, except one 1-valued bit $x_{i}$ where $i \in K$, any other 1 -valued bit $x_{i}$ where $i \in I \cup J \cup K$ must be flipped from 1 to 0 , and $x_{1}$ and $x_{2}$ must be flipped from 0 to 1 . Thus at least $\frac{n}{4}$ bits must be flipped. The probability of this event happening is at most $O\left(n^{-\frac{n}{4}}\right)$.

5) $\mathbf{y}$ is infeasible such that $v(\mathbf{x})<\frac{1}{4\left(1+n^{-4}\right)}$, that means, $\mathbf{y}$ is $\vec{x}_{\text {vio3 }}$.

In this case, any 1 -valued bit $x_{i}$ where $i \in I \cup K$ must be flipped from 1 to 0 , and $x_{1}$ and $x_{2}$ must be flipped from 0 to 1 . Thus at least $\frac{n}{4}$ bits must be flipped. The probability of this event happening is at most $O\left(n^{-\frac{n}{4}}\right)$.

6) $\mathbf{y}$ is infeasible such that $v(\mathbf{x})>\frac{1}{4\left(1+n^{-4}\right)} \cdot \mathbf{y}$ will not be selected since it is dominated by $\vec{x}_{\text {vio1 } 1}$.

From the above analysis, we observe that the probability of generating a non- $\vec{x}_{\text {loc }}$ and non- $\vec{x}_{\text {viol }}$ child and selecting it to the next generation population is small, that is $O\left(n^{-\frac{n}{4}}\right)$.

Next we analyse the role of using a population. Consider the event that the next generation population includes a non- $\vec{x}_{\text {loc }}$ or non- $\vec{x}_{\text {vio1 }}$ child. Since $N$ parents are mutated independently, the probability of the event happening is at most $N O\left(n^{-\frac{n}{4}}\right)$. This implies that the expected number of generations for the EA to reach $\vec{x}_{\max }$ is at least $\frac{1}{N} \Omega\left(n^{\frac{n}{4}}\right)$. Since there are $N$ fitness evaluations at each generation, the expected number of fitness evaluations is $\Omega\left(n^{\frac{n}{4}}\right)$. The required conclusion is proven.

The above theorem shows that the MOEA with the greedy search initialisation finds a $\left(\frac{1}{2}+\frac{1}{n}+\frac{1}{2 n^{3}}\right)$-approximation solution in $\Omega\left(n^{\frac{n}{4}}\right)$ running time. As $n \rightarrow+\infty$, the approximation ratio goes towards $1 / 2$. In other words, the MOEA doesn't substantially improve the solution quality since the greedy search already produces a $1 / 2$ approximation solution during initialisation.

\section{Conclusions}

This paper has assessed the solution quality of an existing MOEA [9] for solving the 0-1 knapsack problem. The solution quality of an EA is measured in terms of the approximation ratio. Two different initialization methods are analysed in the MOEA: local search initialisation and greedy search initialisation. 
When the initial population is produced by the local search, the solution quality of the MOEA might be arbitrarily bad in some instance. That is, given any constant $\alpha \in(0,1)$, the MOEA needs $\Omega\left(n^{\frac{\alpha n}{2}}\right)$ running time to find an $\alpha$-approximation solution in the worst case in some instance.

When the initial population is produced by the greedy search, the MOEA may guarantee a 1/2-approximation solution within polynomial time. However, this improvement is caused by the use of the greedy search, rather than the MOEA itself. In some instance, the MOEA with the greedy search initialisation needs $\Omega\left(n^{\frac{n}{4}}\right)$ running time to find a $\left(\frac{1}{2}+\frac{1}{n}+\frac{1}{2 n^{3}}\right)$-approximation solution. In other words, the MOEA doesn't substantially improve the solution quality comparing with the greedy search.

Other types of initialisation, such as random initialisation, are not considered in the current paper. It is left for future work.

\section{REFERENCES}

[1] S. J. Louis and G. Rawlins, "Pareto optimality, GA-easiness and deception," in Proc. of 5th Inter. Conference on Genetic Algorithms. Morgan Kaufmann, 1993, pp. 118-123.

[2] J. D. Knowles, R. A. Watson, and D. W. Corne, "Reducing local optima in single-objective problems by multi-objectivization," in Evolutionary MultiCriterion Optimization. Springer, 2001, pp. 269-283.

[3] M. T. Jensen, "Helper-objectives: Using multi-objective evolutionary algorithms for single-objective optimisation," Journal of Mathematical Modelling and Algorithms, vol. 3, no. 4, pp. 323-347, 2005.

[4] F. Neumann and I. Wegener, "Minimum spanning trees made easier via multi-objective optimization," Natural Computing, vol. 5, no. 3, pp. 305-319, 2006.

[5] F. Neumann, "Expected runtimes of a simple evolutionary algorithm for the multi-objective minimum spanning tree problem," European Journal of Operational Research, vol. 181, no. 3, pp. 1620-1629, 2007.

[6] T. Friedrich, J. He, N. Hebbinghaus, F. Neumann, and C. Witt, "Approximating covering problems by randomized search heuristics using multi-objective models," Evolutionary Computation, vol. 18, no. 4, pp. 617-633, 2010.

[7] C. Segura, C. A. C. Coello, G. Miranda, and C. León, "Using multi-objective evolutionary algorithms for single-objective optimization," 4OR, vol. 11, no. 3, pp. 201-228, 2013

[8] Y. Zhou, Y. Li, J. He, and L. Kang, "Multi-objective and MGG evolutionary algorithm for constrained optimisation," in Proceedings of 2003 IEEE Congress on Evolutionary Computation. Canberra, Australia: IEEE Press, 2003, pp. 1-5.

[9] Z. Cai and Y. Wang, "A multiobjective optimization-based evolutionary algorithm for constrained optimization," IEEE Transactions on Evolutionary Computation, vol. 10, no. 6, pp. 658-675, 2006.

[10] Y. Wang, Z. Cai, G. Guo, and Y. Zhou, "Multiobjective optimization and hybrid evolutionary algorithm to solve constrained optimization problems," IEEE Transactions on Systems, Man, and Cybernetics, Part B, vol. 37, no. 3, pp. 560-575, 2007.

[11] Y. Wang, Z. Cai, Y. Zhou, and W. Zeng, "An adaptive tradeoff model for constrained evolutionary optimization," IEEE Transactions on Evolutionary Computation, vol. 12 , no. 1 , pp. 80-92, 2008.

[12] L. Jiao, L. Li, R. Shang, F. Liu, and R. Stolkin, "A novel selection evolutionary strategy for constrained optimization," Information Sciences, vol. 239, pp. 122-141, 2013.

[13] Y. Wang and Z. Cai, "Combining multiobjective optimization with differential evolution to solve constrained optimization problems," IEEE Transactions on Evolutionary Computation, vol. 16, no. 1, pp. 117-134, 2012.

[14] _ , "A dynamic hybrid framework for constrained evolutionary optimization," IEEE Transactions on Systems, Man, and Cybernetics, Part B: Cybernetics, vol. 42, no. 1, pp. 203-217, 2012.

[15] T. Friedrich, P. Oliveto, D. Sudholt, and C. Witt, "Analysis of diversity-preserving mechanisms for global exploration," Evolutionary Computation, vol. 17, no. 4, pp. 455-476, 2009.

[16] P. S. Oliveto, J. He, and X. Yao, "Analysis of the (1+1)-EA for finding approximate solutions to vertex cover problems," IEEE Transactions on Evolutionary Computation, vol. 13, no. 5, pp. $1006-1029,2009$.

[17] X. Lai, Y. Zhou, J. He, and J. Zhang, "Performance analysis of evolutionary algorithms for the minimum label spanning tree problem," IEEE Transactions on Evolutionary Computation, vol. 18, no. 6, pp. 860-872, 2014.

[18] S. Martello and P. Toth, Knapsack problems: algorithms and computer implementations. J. Wiley \& Sons, 1990.

[19] H. Kellerer, U. Pferschy, and D. Pisinger, Knapsack Problems. Springer, 2004.

[20] Z. Michalewicz and J. Arabas, "Genetic algorithms for the 0/1 knapsack problem," in Methodologies for Intelligent Systems. Springer, 1994, pp. 134-143.

[21] Z. Michalewicz, Genetic Algorithms + Data Structures = Evolution Programs, 3rd ed. New York: Springer Verlag, 1996.

[22] R. Kumar and N. Banerjee, "Analysis of a multiobjective evolutionary algorithm on the 0-1 knapsack problem," Theoretical Computer Science, vol. 358, no. 1, pp. 104-120, 2006.

[23] Y. Zhou and J. He, "A runtime analysis of evolutionary algorithms for constrained optimization problems," IEEE Transactions on Evolutionary Computation, vol. 11, no. 5, pp. 608-619, 2007.

[24] D. P. Williamson and D. B. Shmoys, The Design of Approximation Algorithms. Cambridge University Press, 2011. 